\title{
Tellurium in a twist
}

\author{
Jim Ibers takes a look at the intriguing structures and bonding found in tellurium and its compounds, and \\ considers their uses in a diversity of fields ranging from metallurgy to electronics.
}

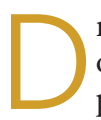
ramatic changes in physical properties occur on descending group 16 of the periodic table. Oxygen, of course, is a gas, polonium a metal, and the in-between elements, sulfur, selenium and tellurium, are all solids that show increasing metallic properties. In fact, tellurium (Te), which I find to be the most interesting of the group, acts very much like most metals.

Tellurium is not an abundant metal. Present at about $1 \mathrm{ppb}$ in the Earth's crust, it is less abundant than gold, platinum or the so-called 'rare-earth' elements. So why should one care about such an obscure element? One answer, of course, is 'because it is there', if one can borrow the phrase that George Mallory used to justify climbing Everest. However, in these days, where instant gratification of those who provide the funds is advisable, other reasons include important current industrial applications and the potential for more. For such applications to be realized, tellurium must be available for basic research in the laboratory and for use in industry. That leads us to a discussion of its sources.

Mining solely for tellurium is not profitable because it is rare and so widely distributed. However, it is most often found in nature in combination with gold, silver, copper and other commercially valuable metals. In fact, gold tellurides are the most common gold-containing minerals. As an aside, tellurium was discovered in 1782 by the Austrian mineralogist Franz Josef Müller von Reichenstein in gold taken from a mine in Hungary. Consequently, tellurium is a by-product in the refining of these other metals. The major source is in the anode sludges produced during the electrolytic refining of copper. These sludges average about $2 \mathrm{wt} \%$ Te. In 2007, total world production of tellurium was about 500 metric tons - a significant increase over previous years. Moreover, as demand for tellurium has increased, so has its cost.
Basic research into the synthesis, structures and properties of telluriumcontaining compounds has increased markedly in recent years. As one measure, we can consider Tecontaining compounds that have been structurally characterized, most often by $\mathrm{X}$-ray diffraction techniques. In the past decade the number of inorganic Te compounds has increased by about $40 \%$; the increase in organic and organometallic Te compounds is nearly $70 \%$. This last figure is surprising given that tellurium is not an essential element for living systems and that many of the synthons and products in organotellurium chemistry make their sulfur analogues smell like roses!

From a personal perspective, when we started our research into ternary solid-state tellurides about 25 years ago it was not known whether these compounds would resemble ternary selenides or not. Some do and some do not. Dissimilarity even extends to the binaries. For example, $\mathrm{NaTe}$ exists whereas NaSe is not known. Ask a first-year chemistry student to describe the structure and formal oxidation states of $\mathrm{NaTe}$ - the answer is that the well-known propensity of sulfur, selenium and tellurium, as opposed to oxygen, to form chains and rings reaches its extreme at tellurium. $\mathrm{NaTe}$ is, in fact, $\mathrm{Na}_{6} \mathrm{Te}\left(\mathrm{Te}_{5}\right)$, which contains the $\mathrm{Na}^{+}$cation together with the $\mathrm{Te}^{2-}$ and the Z-shaped $\mathrm{Te}_{5}{ }^{4-}$ anions. This Z-shaped anion possesses Te-Te distances of 2.82 and $3.08 \AA$, and therein lies one of the exciting differences between Te and S. Structurally, the mineral pyrite (sometimes called 'fool's gold' owing to its golden lustre), of formula $\mathrm{FeS}_{2}$, possesses $\mathrm{S}$ atoms separated by about $2.08 \AA$, the length of a S-S single-bond. The formal oxidation state of Fe is thus +II, not $+\mathrm{IV}$ as one might conclude from the formula. In fact, in essentially all solid-state compounds in which S-S bonding occurs, the $S$ atoms are separated by single bonds. In contrast, in solid-state compounds where there is $\mathrm{Te}-\mathrm{Te}$ bonding, in addition to normal Te-Te single bonds about $2.74 \AA$ in length, there may well be Te-Te separations of up to 3.6 A or more, but still less than the van der Waals separation of $4.1 \AA$. This often makes the assignment of formal oxidation states impossible. The only allotrope of tellurium that is stable at room temperature is an infinite helix (pictured). In that helix, the TeTe distances are about $2.83 \AA$, but there are some short nearest-neighbour interactions at $3.49 \AA$ A. The bonding in Te-containing solidstate compounds, which has engendered numerous theoretical studies, is far more interesting than it is in its lighter congeners.

The major industrial use of tellurium is in metallurgy, where it is an important alloying agent. It is added to steel and to copper to make them more machinable, to cast iron to minimize heat shock, and to lead to reduce fatigue. Another significant industrial use of tellurium is as an accelerator and vulcanizer in the rubber industry. These uses do not require high-purity tellurium. However, the electronics industry increasingly makes use of high-purity tellurium in a variety of new and evolving applications. For example, tellurium is used in the newly developed phase-change memory chips as well as in rewritable CD, DVD and Blu-ray discs. Bismuth telluride is widely used in thermoelectric cooling devices. Such devices find many applications in electronics and consumer products, the latest being their increased use for portable food coolers and, believe it or not, even for car-seat cooling systems. Perhaps the greatest impact of tellurium on our lives might come from cadmium telluride solar panels - still at an early stage, they are among the most efficient for electric power generation. It is possible that tellurium may yet save mankind!

JIM IBERS is in the Department of Chemistry at Northwestern University in the USA. e-mail: ibers@chem.northwestern.edu
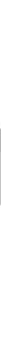